\title{
Experimental study on breakup reaction of beryllium and carbon induced with 14-MeV neutrons based on emitted charged-particle measurements
}

\author{
Keitaro Kondo ${ }^{1, a}$, Isao Murata ${ }^{1}$, Kentaro Ochiai $^{2}$, Hiroyuki Miyamaru ${ }^{1}$, Naoyoshi Kubota ${ }^{2, b}$, Chikara Konno ${ }^{2}$, \\ and Takeo Nishitani ${ }^{2}$ \\ 1 Osaka University, Yamada-oka 2-1, Suita, Osaka 565-0871, Japan \\ 2 Japan Atomic Energy Agency, Shirakata Shirane 2-4, Tokai-mura, Naka-gun, Ibaraki 319-1195, Japan
}

\begin{abstract}
Detailed measurements of $\alpha$-particle emission double differential cross section for beryllium and carbon with $14-\mathrm{MeV}$ incident neutrons were carried out. In order to identify contributing reaction channels to the ${ }^{9} \mathrm{Be}(\mathrm{n}, 2 \mathrm{n}+$ $2 \alpha)$ and ${ }^{12} \mathrm{C}\left(\mathrm{n}, \mathrm{n}^{\prime}+3 \alpha\right)$ reactions, we attempted to reproduce energy distributions of emitted particles by Monte Carlo calculations according to reaction kinematics of a lot of channels which contribute to the reactions. As for the ${ }^{9} \mathrm{Be}(\mathrm{n}, 2 \mathrm{n}+2 \alpha)$ reaction, a contribution of the ${ }^{9} \mathrm{Be}(\mathrm{n}, \alpha){ }^{6} \mathrm{He}$ * channels and the simultaneous breakup channel was suggested to account for the energy and angular distribution of emitted $\alpha$-particles and neutrons. As for the ${ }^{12} \mathrm{C}\left(\mathrm{n}, \mathrm{n}{ }^{\prime}+3 \alpha\right)$ reaction, the ${ }^{12} \mathrm{C}(\mathrm{n}, \alpha){ }^{9} \mathrm{Be} *$ channels were largely contributed and well reproduced the experimental data.
\end{abstract}

\section{Introduction}

Beryllium and carbon are important candidate materials of a fusion reactor. The ${ }^{9} \mathrm{Be}(\mathrm{n}, 2 \mathrm{n}+2 \alpha)$ and ${ }^{12} \mathrm{C}(\mathrm{n}, \mathrm{n}$ ' $+3 \alpha)$ reactions have a relatively large cross section around neutron energy of $14 \mathrm{MeV}$. Double-differential cross section (DDX) of these reactions for emitted neutrons is indispensable to calculate various nuclear properties of the reactor. DDX for emitted $\alpha$-particles is also needed to estimate nuclear heating, material damage and gas production. Mechanism of these reactions is complex because of existence of a lot of reaction channels to reach the 4-body final states of $2 n+2 \alpha$ or $n+3 \alpha$, which are sequential decays accompanied with several intermediate nuclei. Simultaneous breakup further complicates the reaction processes. DDX for emitted neutrons and $\alpha$-particles are closely related with each other because both the particles are emitted through the same reaction. Knowledge for the reactions is hence important in order to evaluate these DDX accurately from engineering requirements. Understanding of the reaction mechanism is also interesting from a viewpoint of nuclear physics.

However, few measurements of DDX for emitted $\alpha$-particles for beryllium and carbon have been carried out so far. Previous analyses for the reaction process were based on insufficient experimental information for emitted $\alpha$-particles, and consistent analyses with emitted neutrons and $\alpha$-particles have been limited [1-3]. We recently developed an improved spectrometer for secondary emitted charged particles using a pencil neutron beam in the Fusion Neutronics Source (FNS) facility in Japan Atomic Energy Agency (JAEA) [4]. Using the spectrometer, we have carried out detailed measurements of $\alpha$-particle emission DDX for beryllium and carbon. The measured $\alpha$-particle DDX data and previous neutron DDX data measured by Takahashi et al. [5] were consistently

\footnotetext{
${ }^{a}$ Presenting author, e-mailktaro@ef. eie.eng.osaka-u.ac.jp

$b$ Present address: Nippon Steel Corporation, 20-1 Shintomi, Futtsu-city, Chiba 293-8511, Japan
}

analyzed considering reaction kinematics in order to investigate channels involved in these reactions.

\section{Experiment}

\subsection{Charged-particle spectrometer using a pencil neutron beam}

All the present measurements of DDX for emitted $\alpha$-particles were carried out with the pencil DT neutron beam available at FNS/JAEA. The use of the neutron beam is essential in order to reduce background neutrons around an experimental apparatus extensively. The details of the measurement technique are described in ref. [4].

A schematic view of the facility and the experimental setup is shown in figure 1 . The facility is an accelerator-based intense neutron source. Generated DT neutrons are collimated by a $2-\mathrm{m}$ thick shielding structure with a narrow hole of $2 \mathrm{~cm}$ in diameter. The mean energy of the neutron beam is 14.2 MeV. A vacuum chamber was set at the outlet of the neutron beam, and a sample material was fixed at the center of the chamber. The sample materials used in the present study are a beryllium foil of $20 \mu \mathrm{m}$ thickness and a carbon foil of $5 \mu \mathrm{m}$ thickness. A counter-telescope system with a pair of silicon surface barrier detectors, one for $\Delta \mathrm{E}$ (thickness of $9.6 \mu \mathrm{m}$ ) and the other for $\mathrm{E}$ (thickness of $760 \mu \mathrm{m}$ ), is employed in order to distinguish kinds of emitted charged particles. The detectable minimum energy of the telescope, which depends on the thickness of the $\Delta \mathrm{E}$ detector, is $2.5 \mathrm{MeV}$ for $\alpha$-particles. In order to lower the detectable energy range, anticoincidence spectra of the $\Delta \mathrm{E}$ detector are utilized. When the $\Delta \mathrm{E}$ detector of $9.6 \mu \mathrm{m}$ thickness is used, the threshold energies beyond which the $\Delta \mathrm{E}$ detector can be penetrated are around $700 \mathrm{keV}$ for protons and around $1.0 \mathrm{MeV}$ for tritons. The anticoincidence spectrum above those threshold energies for protons and tritons hence originates only from $\alpha$ particles or particles heavier than $\alpha$-particles. In the present 


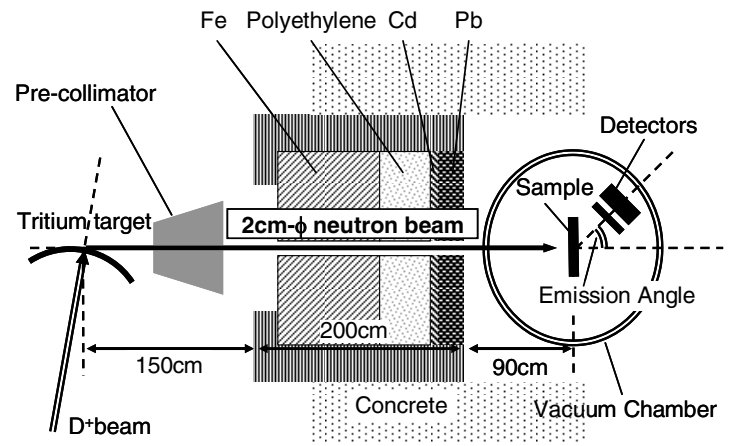

Fig. 1. Schematic view of experimental setup.

measurement, recoiling beryllium or carbon and ${ }^{9} \mathrm{Be}$ particles emitted via the ${ }^{12} \mathrm{C}(\mathrm{n}, \alpha){ }^{9} \mathrm{Be}$ reaction cannot be negligible and their contributions are calculated and subtracted. As a result, the measurement of $\alpha$-particles with a minimum energy of around $1 \mathrm{MeV}$ was successfully realized.

In the present measurement, the incident neutron fluence was determined by the aluminum foil activation method. For a standard cross section, $122.0 \mathrm{mb}$ for the ${ }^{27} \mathrm{Al}(\mathrm{n}, \alpha)$ reaction evaluated in JENDL-3.3 [6] was used.

\subsection{Data analysis}

In order to obtain an actual energy spectrum of emitted charged particles, the measured spectrum must be corrected for energy loss in the sample. A relationship between the actual spectrum and the measured spectrum was calculated by the Monte Carlo code SRIM-2003 [7] combined with pre- and post-processing codes we made. Then the spectrum unfolding was carried out with our original code based on the spectrum type Bayes estimation method [8] to obtain the actual spectrum.

\section{Results}

\subsection{Measurement for beryllium}

DDX data for the ${ }^{9} \mathrm{Be}(\mathrm{n}, \mathrm{x} \alpha)$ reaction were obtained at 9 emission angles from $15 \mathrm{deg}$ to $135 \mathrm{deg}$. There has been an only previous measurement by Ferenc et al. [9], which was, however, a relative measurement and measured energy range was not so wide. Hence, our data is the only existing detailed experiment. Figures 2 and 3 show the obtained DDX at 20 and 40deg and comparison with evaluated nuclear data taken from JENDL-3.3 and ENDF/B-VI.8 [10]. In the measured DDX, two peaks corresponding to the ${ }^{9} \mathrm{Be}\left(\mathrm{n}, \alpha_{0}\right)^{6} \mathrm{He}_{\text {(ground state) }}$ and ${ }^{9} \mathrm{Be}\left(\mathrm{n}, \alpha_{1}\right){ }^{6} \mathrm{He}^{*}(1.8 \mathrm{MeV})$ reactions are identified. The evaluated libraries significantly underestimate the higher energy part of DDX at $20 \mathrm{deg}$ while these well agree with each other at $40 \mathrm{deg}$. We should investigate the mechanism to account for this strong $\alpha$-particle emission of higher energy at forward angles.

\subsection{Measurement for carbon}

DDX data for the ${ }^{12} \mathrm{C}(\mathrm{n}, \mathrm{x} \alpha)$ reaction were obtained at 10 emission angles from $15 \mathrm{deg}$ to $150 \mathrm{deg}$. Figure 4 shows the

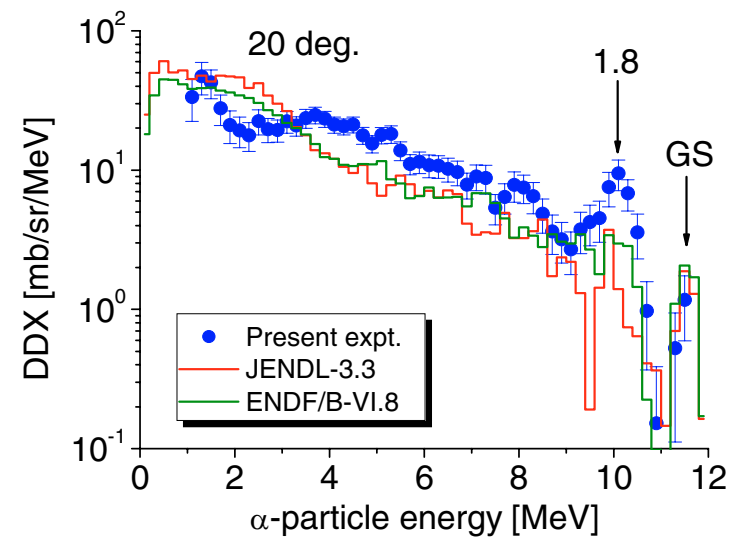

Fig. 2. Measured $\mathrm{DDX}$ for ${ }^{9} \mathrm{Be}(\mathrm{n}, \mathrm{x} \alpha)$ reaction at emission angle of $20 \mathrm{deg}$ and comparison with evaluated nuclear data.

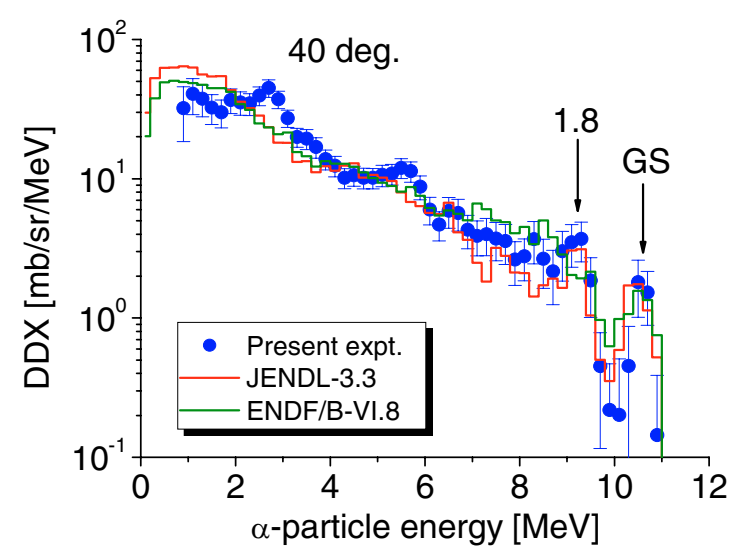

Fig. 3. Measured $\mathrm{DDX}$ for ${ }^{9} \mathrm{Be}(\mathrm{n}, \mathrm{x} \alpha)$ reaction at emission angle of $40 \mathrm{deg}$ and comparison with evaluated nuclear data.

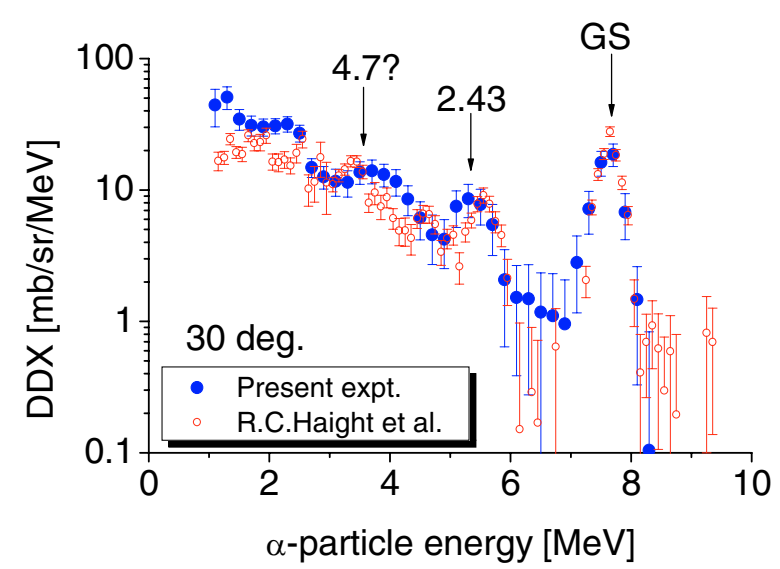

Fig. 4. Measured DDX for ${ }^{12} \mathrm{C}(\mathrm{n}, \mathrm{x} \alpha)$ reaction at emission angle of $30 \mathrm{deg}$ and comparison with previous measurement.

obtained DDX at $30 \mathrm{deg}$ and comparison with the previous data measured by Haight et al. [11]. There is no evaluated DDX which can be compared with our data directly. In our obtained DDX, obvious peaks corresponding to the ${ }^{12} \mathrm{C}\left(\mathrm{n}, \alpha_{0}\right){ }^{9} \mathrm{Be}_{(\text {Ground State })}$ and ${ }^{12} \mathrm{C}(\mathrm{n}, \alpha){ }^{9} \mathrm{Be}^{*}(2.43 \mathrm{MeV})$ reactions are clearly identified. The contribution of ${ }^{9} \mathrm{Be}^{*}(4.7 \mathrm{MeV})$ might exist. Between both the measurements, agreement of the higher energy part is fairly good, while a slight discrepancy 


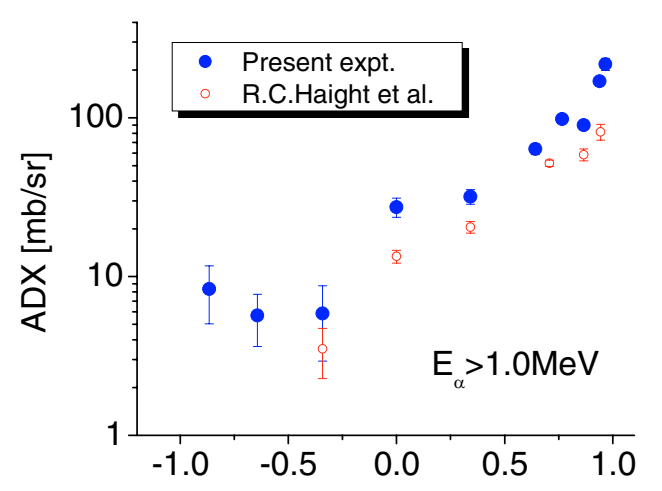

Emission angle in laboratory-system [cos unit]

Fig. 5. Measured $\mathrm{ADX}$ for ${ }^{12} \mathrm{C}(\mathrm{n}, 3 \alpha)$ n' reaction and comparison with previous measurement, where contribution of ${ }^{12} \mathrm{C}\left(\mathrm{n}, \alpha_{0}\right)$ reaction was excluded.

was observed below $3 \mathrm{MeV}$. Figure 5 shows the angulardifferential cross section $(\mathrm{ADX})$ for the ${ }^{12} \mathrm{C}\left(\mathrm{n}, \mathrm{n}^{\prime}+3 \alpha\right)$ reaction, where the contribution of the ${ }^{12} \mathrm{C}\left(\mathrm{n}, \alpha_{0}\right)$ is excluded. There is a strongly forward-peaked and slightly fluctuated distribution, which would suggest some direct reaction contribution. The difference between the present experiment and Haight's measurement is caused by the discrepancy of DDX below $3 \mathrm{MeV}$.

\section{Analysis based on reaction kinematics}

\subsection{Monte Carlo calculation}

We tried to identify the reaction channels which contribute to the ${ }^{9} \mathrm{Be}(\mathrm{n}, 2 \mathrm{n}+2 \alpha)$ and ${ }^{12} \mathrm{C}\left(\mathrm{n}, \mathrm{n}^{\prime}+3 \alpha\right)$ reactions. For this purpose, emitted energy distributions of all possible channels both for $\alpha$-particles and for neutrons were calculated according to reaction kinematics by the Monte Carlo method. The calculated spectra were fitted to experimental data by the least-squares method and the contributing channels were determined. For the experimental data, our measuring DDX for $\alpha$-particles and DDX for neutrons measured by Takahashi et al. [5] were used. The calculation procedure was the same as that is ref. [1]. In the calculation, simply 2-body sequential decays via excited states of ${ }^{12} \mathrm{C},{ }^{9} \mathrm{Be}$ and ${ }^{6} \mathrm{He}$ and the 3-body simultaneous breakup with a pure phase space were considered. Decay modes for ${ }^{8} \mathrm{Be}^{*}$ and ${ }^{5} \mathrm{He} *$ from the excited nuclei were adopted according to refs. [2,12,13]. For all the intermediate states, the density-of-states function was given by the Breit-Wigner distribution with constant level widths evaluated in refs. $[12,13]$. The angular distributions for the inelastic scattering of neutrons, which were given in the calculation, were cited from ref. [5] for the excited states of ${ }^{9} \mathrm{Be}$ at $2.43,4.7,6.76$ and $7.94 \mathrm{MeV}$ and ${ }^{12} \mathrm{C}$ at 7.65 and $9.64 \mathrm{MeV}$. For the other excited states of ${ }^{9} \mathrm{Be}$ and ${ }^{12} \mathrm{C}$, isotropic distribution in center-of-mass system was assumed. In other 2-body decays, isotropic distribution in center-of-mass system was also assumed.

\subsection{Beryllium}

Figure 6 shows the calculated DDX of the ${ }^{9} \mathrm{Be}(\mathrm{n}, 2 \mathrm{n}+2 \alpha)$ reaction both for emitted $\alpha$-particles and for neutrons at
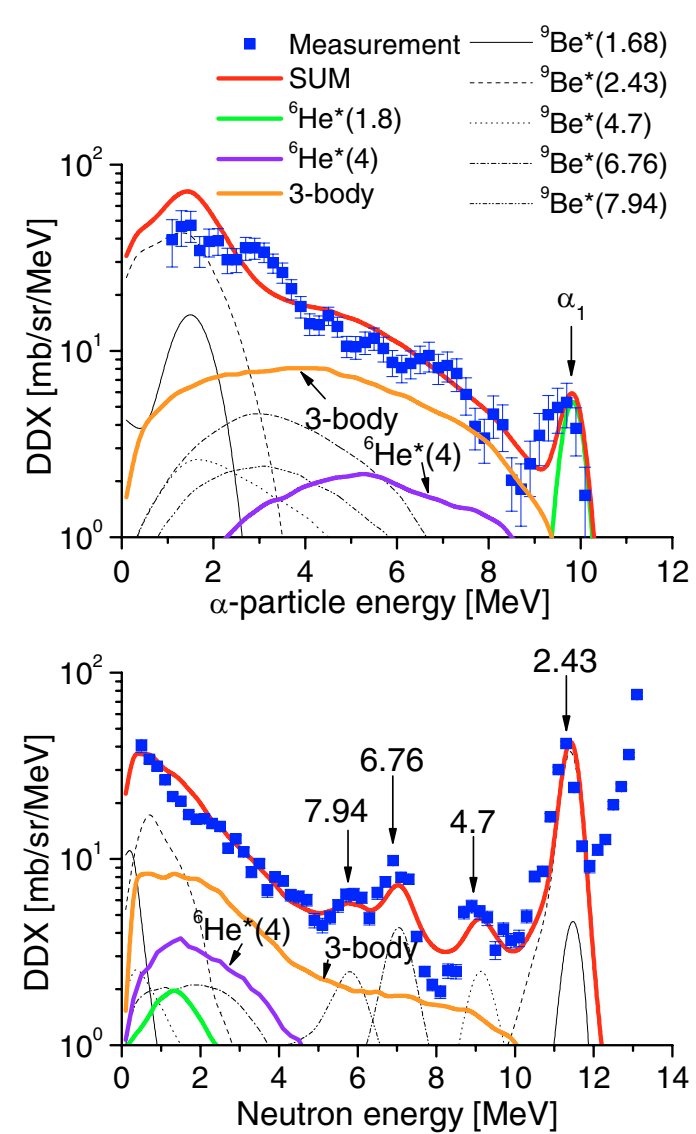

Fig. 6. Measured and calculated DDX of ${ }^{9} \mathrm{Be}(\mathrm{n}, 2 \mathrm{n}+2 \alpha)$ reaction at emission angle of $30 \mathrm{deg}$ both for $\alpha$-particles and for neutrons.

$30 \mathrm{deg}$. The component indicated as " 3 -body" in the figure is contribution of the ${ }^{9} \mathrm{Be}\left(\mathrm{n}, \mathrm{n}^{\prime}+\alpha+{ }^{5} \mathrm{He}\right)$ simultaneous breakup reaction. The 3-body and ${ }^{9} \mathrm{Be}(\mathrm{n}, \alpha){ }^{6} \mathrm{He}^{*}(\geq 1.8 \mathrm{MeV})$ channels are essential to reproduce the experimental results well. In the present study, the contribution of the ${ }^{9} \mathrm{Be}(\mathrm{n}, \alpha)^{6} \mathrm{He}^{*}$ channels was estimated as around $15 \%$ and the contribution of the simultaneous breakup channel was estimated as around $30 \%$.

\subsection{Carbon}

Figure 7 shows the calculated DDX of the ${ }^{12} \mathrm{C}\left(\mathrm{n}, \mathrm{n}{ }^{\prime}+3 \alpha\right)$ reaction both for emitted $\alpha$-particles and for neutrons at $30 \mathrm{deg}$. In the present estimation, the ${ }^{9} \mathrm{Be}^{*}(2.43 \mathrm{MeV})$ and ${ }^{9} \mathrm{Be}^{*}(4.7 \mathrm{MeV})$ channels play an important role. The continuum in lower energy of the neutron DDX is reproduced well. These channels would be reasonable to account the strong forward-peaked distribution. The contribution of the ${ }^{12} \mathrm{C}(\mathrm{n}, \alpha){ }^{9} \mathrm{Be}^{*}{ }_{(2.43 \mathrm{MeV})}$ and the ${ }^{12} \mathrm{C}(\mathrm{n}, \alpha){ }^{9} \mathrm{Be}^{*}{ }_{(4.7 \mathrm{MeV})}$ channels was estimated as around $25 \%$ and $10 \%$, respectively.

\section{Summary}

We have conducted detailed measurements of $\alpha$-particle emission DDX for beryllium and carbon using our developed spectrometer with a pencil DT neutron beam. In order to identify the reaction channels which contribute to the 

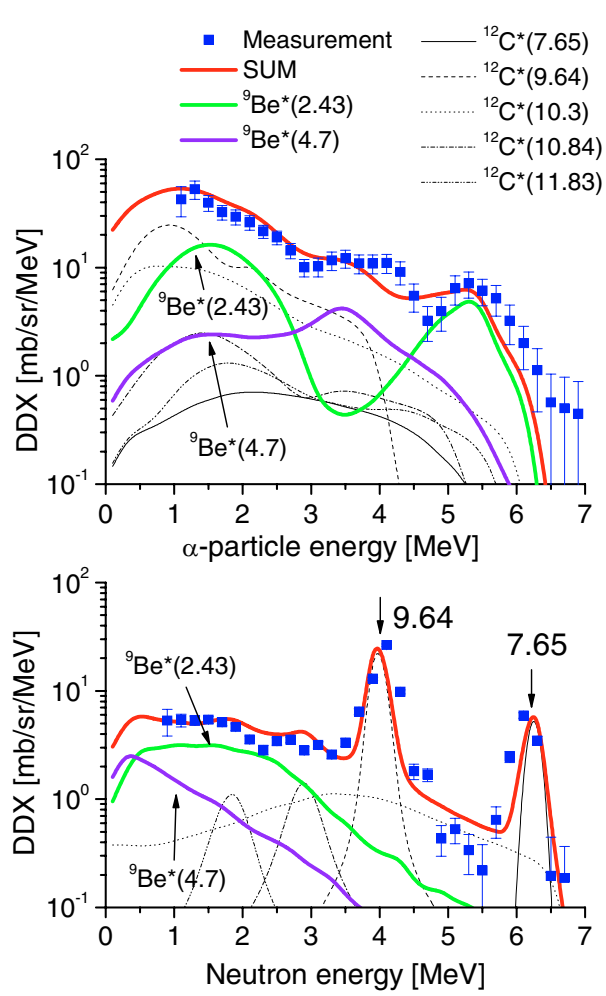

Fig. 7. Measured and calculated $\mathrm{DDX}$ of ${ }^{12} \mathrm{C}\left(\mathrm{n}, \mathrm{n}^{\prime}+3 \alpha\right)$ reaction at emission angle of $30 \mathrm{deg}$ both for $\alpha$-particles and for neutrons.

${ }^{9} \mathrm{Be}(\mathrm{n}, 2 \mathrm{n}+2 \alpha)$ and ${ }^{12} \mathrm{C}\left(\mathrm{n}, \mathrm{n}^{\prime}+3 \alpha\right)$ reactions, emitted energy distributions of all possible channels both for $\alpha$-particles and for neutrons were calculated according to reaction kinematics. The calculated spectra were fitted to our measuring DDX for $\alpha$-particles and DDX for neutrons measured by Takahashi et al. As a result, the information of contributing channels was obtained. As for the ${ }^{9} \mathrm{Be}(\mathrm{n}, 2 \mathrm{n}+2 \alpha)$ reaction, a contribution of the ${ }^{9} \mathrm{Be}(\mathrm{n}, \alpha)^{6} \mathrm{He}^{*}$ and simultaneous breakup channels are essential to account for the measured DDX of emitted $\alpha$-particles. As for the ${ }^{12} \mathrm{C}\left(\mathrm{n}, \mathrm{n}^{\prime}+3 \alpha\right)$ reaction, the ${ }^{12} \mathrm{C}(\mathrm{n}, \alpha){ }^{9} \mathrm{Be}^{*}$ channels are largely contributed and well reproduce the lower energy continuum of neutron DDX. Significant contribution of these first- $\alpha$-emission channels would suggest importance of the $\alpha$-particle knock-on or stripping process. Further theoretical approaches are desired in order to investigate the direct reaction process in these nuclei.

The authors wish to acknowledge the FNS staff for their excellent operation of the FNS accelerator: Mrs. C. Kutsukake, S. Tanaka, Y. Abe, M. Seki, Y. Oginuma and M. Kawabe. The support and encouragement provided by K. Okada, K. Takakura, S. Takagi and S. Shido are also greatly appreciated.

\section{References}

1. S.T. Perkins, E.F. Plechaty, R.J. Howerton, Nucl. Sci. Eng. 90, 83 (1985).

2. B. Antolković, Z. Dolenec, Nucl. Phys. A 237, 235 (1975).

3. B. Antolković, G. Dietze, H. Klein, Nucl. Sci. Eng. 107, 1 (1991).

4. K. Kondo, I. Murata, K. Ochiai et al., Nucl. Instrum. Meth. A 568, 723 (2006).

5. A. Takahashi, E. Ichimura, Y. Sasaki, H. Sugimoto, OKTAVIAN Report, A-87-03, Osaka University, Japan (1987).

6. K. Shibata, T. Kawano, T. Nakagawa et al., J. Nucl. Sci. Technol. 39, 1125 (2002).

7. J.F. Ziegler, J.P. Biersack, The Stopping Power and Ranges of Ions in Matter, Pergamon (New York, 1985); Computer code SRIM-2003, http://www.srim.org/.

8. S. Iwasaki, Reactor Dosimetry (World Scientific Publishing Co. Pte. Ltd., Singapore, 1998) 245.

9. D. Ferenc, B. Antolković, G. Paić et al., Nucl. Sci. Eng. 101, 1 (1989).

10. V. McLane and Members of the Cross section Evaluation Working Group, ENDF-201, ENDF/B-VI Summary Documentation, BNL-NCS-17541, 4th Edition, Suppl. I (1996).

11. R.C. Haight, S.M. Grimes, R.G. Johnson, H.H. Barschall, Nucl. Sci. Eng. 87, 41 (1984).

12. D.R. Tilley, J.H. Kelley, J.L. Godwin et al., Nucl. Phys. A 745, 155 (2004).

13. F. Ajzenberg-Selove, Nucl. Phys. A 506, 1 (1990). 